\title{
Stimulated Brillouin Scattering Threshold in Double-Clad Fibers with various cladding shapes
}

\author{
Luca Tartara ${ }^{1}$, Christophe Codemard ${ }^{2}$, Jean-Noel Maran ${ }^{3}$ \\ 1. Department of Eelectronics, Università degli Studi di Pavia, via Ferrata 1, IT-27100 Pavia, Italy \\ 2.Optoelectronics Research Centre, University of Southampton, Highfield, Southampton SO17 1BJ, United Kingdom \\ 3.Coractive High Tech Inc., 2700, Jean Perrin, Suite 121, Quebec (QC) G2C 1S9, Canada
}

We present a detailed numerical investigation about the stimulated Brillouin scattering (SBS) threshold power of double-clad optical fibers having different inner-cladding shapes. Generally, fibers with broken symmetry inner-cladding are usually employed in fiber lasers and amplifier in order to enhance pump light absorption by the doped core [1]. The acoustic response of such geometries is not known because their numerical characterizations are complicated. Even more difficulties occur when the fiber possess an acoustic antiwaveguide core since in that case the Brillouin response is often ruled by very high-order modes [2].

We have recently developed a numerical tool which does not rely on any kind of symmetry of the fiber structure and is able to compute all the supported acoustic modes [3] in any arbitrary geometry. Here, we apply this tool to investigate the effect of the cladding shape of fiber on the SBS threshold power.

In order to highlight the impact of the geometry, we have kept fixed the inner cladding area so that the amount of material and the number of modes stays the same. Our reference fiber is a circularly symmetric fiber with an inner cladding area fixed to $7088 \mu \mathrm{m}^{2}$ corresponding to a $95 \mu \mathrm{m}$ diameter cladding. Its $5-\mu \mathrm{m}$ core is a step index with a $0.2 \mathrm{NA}$ behaving as an acoustic waveguide, i.e. a fiber for which the acoustic velocity is higher in the inner cladding than in the core. All the other fibers considered have an acoustic anti-waveguiding core with the two values of the acoustic velocity being reversed. Table 1 summarizes the results.

\begin{tabular}{|c|c|c|}
\hline Core & $\begin{array}{c}\text { Cladding shape } \\
\text { (dimension) }\end{array}$ & $\begin{array}{c}\text { Threshold } \\
\text { increase } \\
\text { [dB] }\end{array}$ \\
\hline acoustic waveguide (a) & circular $(\varnothing 95 \mu \mathrm{m})$ & 0 \\
\hline anti-waveguide & circular $(\varnothing 95 \mu \mathrm{m})$ & 7.2 \\
\hline $\begin{array}{c}\text { offset }(50 \%) \\
\text { anti-waveguide }\end{array}$ & circular $(\varnothing 95 \mu \mathrm{m})$ & 6.9 \\
\hline anti-waveguide & D-shape $(5 \%)$ & 6.8 \\
\hline anti-waveguide & Double D-shape $(5 \% / 8 \%)$ & 11.5 \\
\hline anti-waveguide & elliptical $(67.5 \times 135 \mu \mathrm{m})$ & 10.8 \\
\hline anti-waveguide & hexagonal & 9.1 \\
\hline anti-waveguide & octagonal & 8.1 \\
\hline anti-waveguide (b) & rectangular $(60 \mathrm{x} 120 \mu \mathrm{m})$ & 11.7 \\
\hline anti-waveguide & square $(85 \mu \mathrm{m})$ & 9.1 \\
\hline
\end{tabular}

Table 1 Threshold increase for double-clad fibers having different inner-cladding shapes.

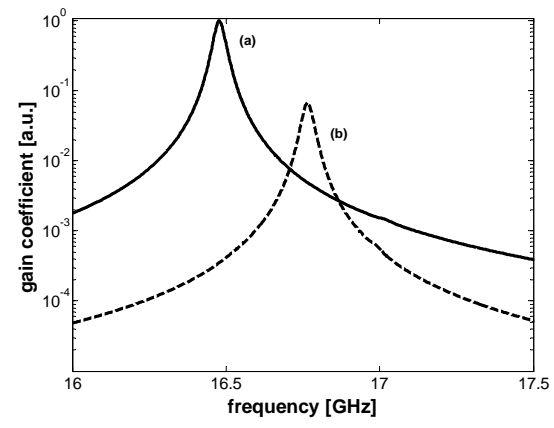

Fig. 1 SBS gain spectrum for an acoustic waveguiding fiber with circular cladding (a) and for an anti-acoustic waveguiding fiber with a rectangular cladding (b).

The results clearly demonstrate a significative impact of the inner-cladding shape on the SBS threshold of the antiwaveguide core. In quasi-circular cladding shape such as the D-Shaped, the offset core, hexagonal, etc ..., the SBS threshold mostly remains unaffected. By contrast, claddings with strong deformation and where the symmetry is broken exhibit a higher increase of the SBS threshold power.

Although these results concern fibers with a relatively small cladding size compared to that used in fiber laser, we believe that such fiber can have application in lower power system e.g. amplifier or for passive fiber e.g. in telecom systems.

\section{References}

[1] P. Even and D. Pureur, "High Power Double Clad Fiber Lasers: a Review", Proc. SPIE 4638, 1 (2002).

[2] Y. Koyamada, S. Sato, S. Nakamura, H. Sotobayashi, and W. Chujo, "Simulating and Designing Brillouin Gain Spectrum in SingleMode Fibers", J. Lightwave Technol. 22, 631 (2004).

[3] L. Tartara, C. Codemard, J.N. Maran, R. Cherif, and M. Zghal, "Full Modal Analysis of the Brillouin Gain Spectrum of an Optical Fiber", submitted to Opt. Comm. 